\title{
Classification of urban feature from unmanned aerial vehicle images using GASVM integration and multi-scale segmentation
}

\author{
M.Modiri, A.Salehabadi, M.Mohebbi, A.M.Hashemi, M.Masumi \\ National Geographical Organization
}

Ar_mohammadi_hashemi@yahoo.com

KEY WORDS: Classification, UAV images, GASVM, segmentation

\begin{abstract}
:
The use of UAV in the application of photogrammetry to obtain cover images and achieve the main objectives of the photogrammetric mapping has been a boom in the region. The images taken from REGGIOLO region in the province of, Italy Reggio -Emilia by UAV with non-metric camera Canon Ixus and with an average height of 139.42 meters were used to classify urban feature. Using the software provided SURE and cover images of the study area, to produce dense point cloud, DSM and Artvqvtv spatial resolution of $10 \mathrm{~cm}$ was prepared. DTM area using Adaptive TIN filtering algorithm was developed. NDSM area was prepared with using the difference between DSM and DTM and a separate features in the image stack. In order to extract features, using simultaneous occurrence matrix features mean, variance, homogeneity, contrast, dissimilarity, entropy, second moment, and correlation for each of the RGB band image was used Orthophoto area. Classes used to classify urban problems, including buildings, trees and tall vegetation, grass and vegetation short, paved road and is impervious surfaces. Class consists of impervious surfaces such as pavement conditions, the cement, the car, the roof is stored. In order to pixel-based classification and selection of optimal features of classification was GASVM pixel basis. In order to achieve the classification results with higher accuracy and spectral composition informations, texture, and shape conceptual image featureOrthophoto area was fencing. The segmentation of multi-scale segmentation method was used.it belonged class. Search results using the proposed classification of urban feature, suggests the suitability of this method of classification complications UAV is a city using images. The overall accuracy and kappa coefficient method proposed in this study, respectively, 47/93\% and 84/91\% was.
\end{abstract}

\section{Introduction}

The use of UAVs in various fields and particularly photogrammetry has expanded. UAV is the Motor vehicle and are reusable unmanned. Van Blyenburgh, 1999)). This means the remote control, semi-automatic, automatic, or a combination of the ability to differentiate. In UAV with manned aircraft, it is clear that the main difference between the two systems is that the UAV pilot in the aircraft to be operated without physical presence.The major advantages of UAVs compared with manned aircraft systems, including: the UAV can be dangerous situations without endangering human life and can be used in inaccessible areas, the low-altitude flight profile close to objects where the system can not be manned fly as sites of natural disasters, and the volcanic highlands, plains, flood, storm, earthquake, wilderness areas and areas where access is difficult and there Hvads.dr scenes No manned aircraft is not available or will not be allowed to fly UAVs are sometimes the only practical way. Moreover, in overcast 
conditions and relatively rainy climate, data acquisition with UAVs because they are able to fly below the clouds is still possible weather conditions such as data acquisition with integrated large format cameras installed on manned aircraft due to fly height does not allow for more. In addition, one of the main advantages of UAVs lack of psychological pressure or acceleration $G$ and the height of the pilot. In addition, supplementary benefits in the ability and the ability for quick access to information Real time, as long as images and videos can be transmitted in real time to a ground control station. More UAV systems on the market is low-cost systems. as a result one of the main advantages of using UAVs is the cost factor. Figure 1 position among other branches UAV photogrammetric surveying shows.

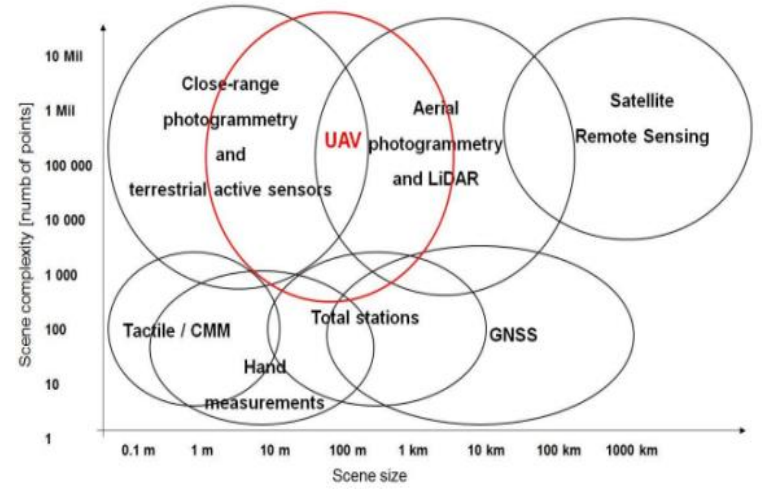

Figure 1. UAV photogrammetry place alongside other sub-branches of photogrammetry

In this study, we tried to classify the urban feature in the images taken by a UAV with the highest precision and quality style. Then, in the next section details the proposed method and the third part of the data used and the desired characteristics of the aircraft will be introduced. Then, in the fourth part of the implementation and results of the evaluation will be presented and discussed. Finally, conclusions and recommendations expressed in the fifth.

\section{The offered method to classify the urban feature of UAV images}

The proposed method to classify urban feature of images captured by the UAV consists of five main parts. In the first part of convergent images taken from the area Orthophoto, DSM, DTM and NDSM is provided. The next section features GLCM of Orthophoto area of tissue matrix is produced and then used as input along with NDSM fall. The third part of the core SVM classifier using genetic algorithms and parameters of the main features of the kernel SVM determined. In fact, in this part of the image classification features and parameters are set using GASVM. In the fourth part Orthophoto of images taken by UAV produced using multi-scale segmentation. Finally in the fifth classification image by image segmentation merge with. This integration is this section that are more than $50 \%$ of classes in the image of Nada floor covering belong to that class.

\subsection{Preparation of Orthophoto, DSM, DTM, NDSM}

Error Orthophoto place UAV sensors made from images taken by the Landsat images have more importance than manned aircraft. Because of the spatial and angular parameters, UAV images of much lower order than aerial imagery that has regular flight routes and distances are given specific shooting. In precision work, photogrammetric UAV, camera stations should be considered so that we can cover all parts necessary for the images to be created. In order to detect and remove systematic errors in each station camera takes multiple images from different angles. This will complicate the basic design and features of its mathematical model. Amhar in 1988 suggested that the use of DSM produced Orthophoto right. Barazzetti and co-produced Orthophoto of images taken by 
a UAV sensor consists of three main stages were as follows:

- justification Images

- Production of the grid

- Production Orthophoto Field

The justification images, using automatic matching algorithm such as SIFT and SURF cloud common condition in images produced by the linear adjustment beam out images of justification is provided. It should be noted that elements of internal justification and calibration method is produced by the UAV camera calibration parameters. In the second phase for the production of grid fitted sheet on the corresponding point cloud generated in the previous step and therefore DSM production area to be used. DTM production area of Adaptive TIN filtering method is used.

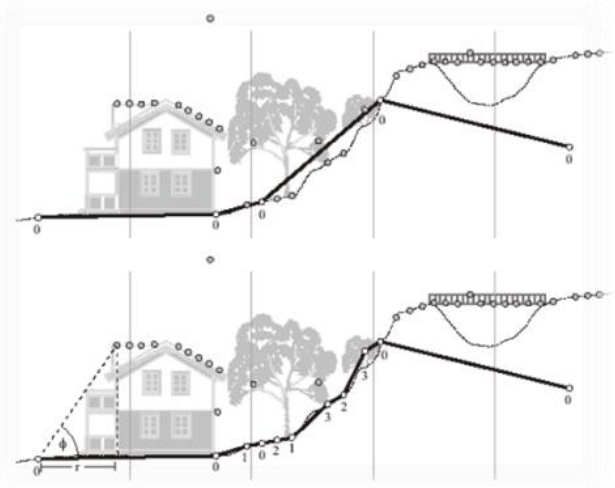

Figure 2. Progressive compression algorithm Axelsson. first estimate based on the minimum height of the bare ground in neighborhoods (above) and subsequent estimates based on the angle and distance to the nearest node

Labeling function for this algorithm is as follows

$$
\phi\left(V_{i}\right)=\left\{\begin{array}{lr}
1 & v_{i} \in \text { THN } \\
0 & \text { ELSE }
\end{array}\right.
$$

The main feature of this algorithm, the ability of the algorithm to model the uneven levels of returns, in addition to the significant potential of urban areas to separate from the rest of the raw LiDAR is the bare ground. In this study was to identify the feature high model surface normal level nDSM production. Then by applying a threshold elevation on the high feature identification was done. Digital models are used to produce normal levels of the following equation.

\section{2. extraction of texture features}

Using a combination of spectral and textural classes with similar spectral responses (such as low density vegetation areas and urban areas) increased the classification accuracy (Zhang, 1999). Feature extraction involves extracting tissue characteristics of spectral bands. One of the common methods used to extract texture features matrix images at the same event. Haralick and colleagues in 1973 had taken images at the same time features based on occurrence matrix and each was introduced as a representative of a characteristic image (Haralick et al., 1973). In this study the properties of mean, variance, homogeneity, contrast, dissimilarity, entropy, second moment, and correlation were used.

\section{3- Classification by GASVM}

Goldenberg in 1989. The framework and rules on genetic algorithms introduced in 1990, proved the convergence of genetic algorithms (Goldberg, 1989).is. Finding an optimal subset of features, directly related to the choice of the fitness function (Kyavrz, 1386). Which amounts to a subset of their various merit functions, are different. In this study, the following criteria in order to select the optimal SVM classification kappa coefficient was used. Classification support vector machines (SVM) is a form of supervised classification was introduced in 1965 by Vapnik [11]. Many studies have shown that this classification of other parametric classification methods such as Artificial Neural Networks (MLP) and 
parametric methods such as classification Maximum likelihood is the more accurate [12]. SVM classification of certain statistical distribution of data does not assume and because remote sensing data usually have no statistical distribution, it is important that the parameters are heterogeneous and also in cases of demonstrated well [13]. The basic idea of SVM finding the optimal hyperplane to separate the two classes are separated by the greatest margin. For the non-linear separation through kernel function input space into a higher dimensional feature space where a linear list can be found above, the mapping.

\subsection{Multi-scale segmentation}

At this stage Orthophoto production of UAV images were segmented. In fact, break the image segmentation polygon areas with different characteristics. The image of the objects are called. Multi-scale segmentation is one of the best methods of segmentation of images. Multi-scale segmentation a segmentation method that integrates areas of a pixel start and for successive pixels or objects merge the existing image. The successive step integration of the region so far-is that the heterogeneity of the parameter in question does not exceed the threshold. This bottom-up approach based on local integration technique progress. The local integration combine objects based on the nearest object and parameter control together the final image does not exist [42]. There is an optimal algorithm for a specified number of objects at least parameter heterogeneity and homogeneity provides maximum parameter. The best method of two-way. Figure 39 -section of the most important methods of display.

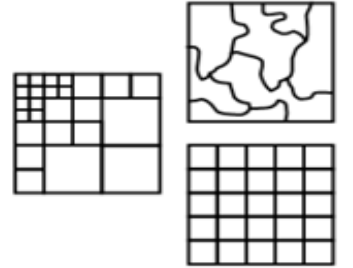

Figure 3 types of fencing, the above figure, multi-scale and left, the four-and-down tree, grid

\subsection{The integration of the results of the classification and segmentation}

At this stage, the results of image segmentation and classification Multiscale segmentation Orthophoto with other UAV images are produced. Each of the segments produced by segmentation Chndmqyas.h that more than $50 \%$ of classes in the cover image classification to belong to that class. So the results of the segmentation and classification, in the final classification maps are very impressive.

\section{3. used Data}

the images which taken from REGGIOLO region in the province of Emilia, Italy Rjyv- by UAV with nonmetric camera Canon Ixus and of medium height $139.42 \mathrm{~m}$ was used to classify urban feature. The study involved unmanned aircraft over the city average of $42 / 139$ meters altitude flying and 18 of the region's image. Using the software provided SURE and cover images of the study area, to produce dense point cloud, DSM and carefully place Artvqvtv $10 \mathrm{~cm}$ was used. Figure 1 Study area and DSM obtained OrthophotoUAV images show. 


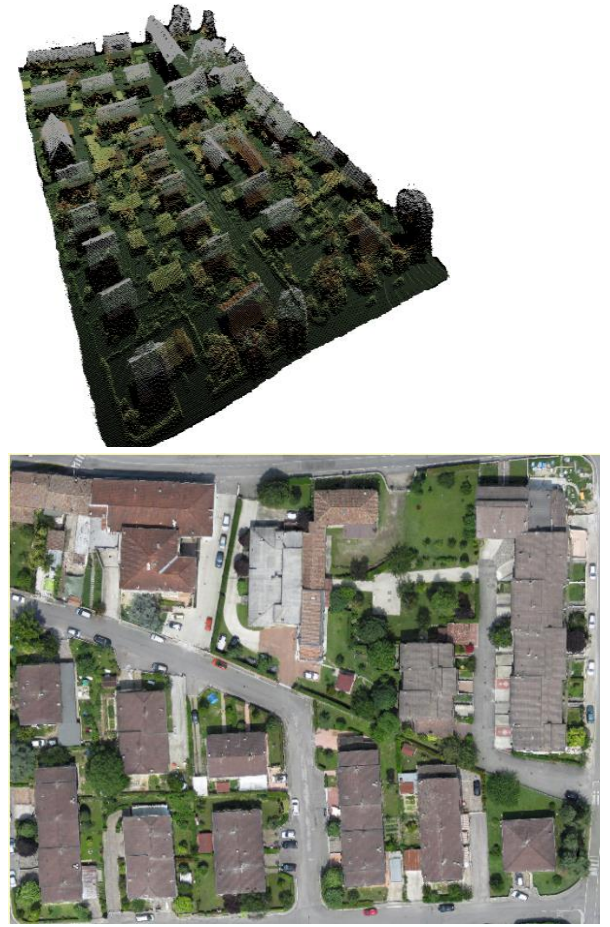

Figure 4. The provided data a) Ortho Photo area

B) produced DSM

Ortho photo and DSM and area DTM as input data are entered into the offered algorithm to classification of urban features.

\section{Implementation and evaluation of results}

In order to implement the proposed method, the software MATLAB 2013b, SURE and eCognition 9.2 is used. Other parts of the proposed method in accordance with the details described in the previous section's programming. This section details the implementation and results presented and discussed.

\subsection{Evaluation Results}

DTM area was provided using Adaptive TIN filtering algorithm. NDSM area using the difference between DSM and DTM was prepared and a separate features in the image stack. Figure 6, DTM and NDSM of the area shows.

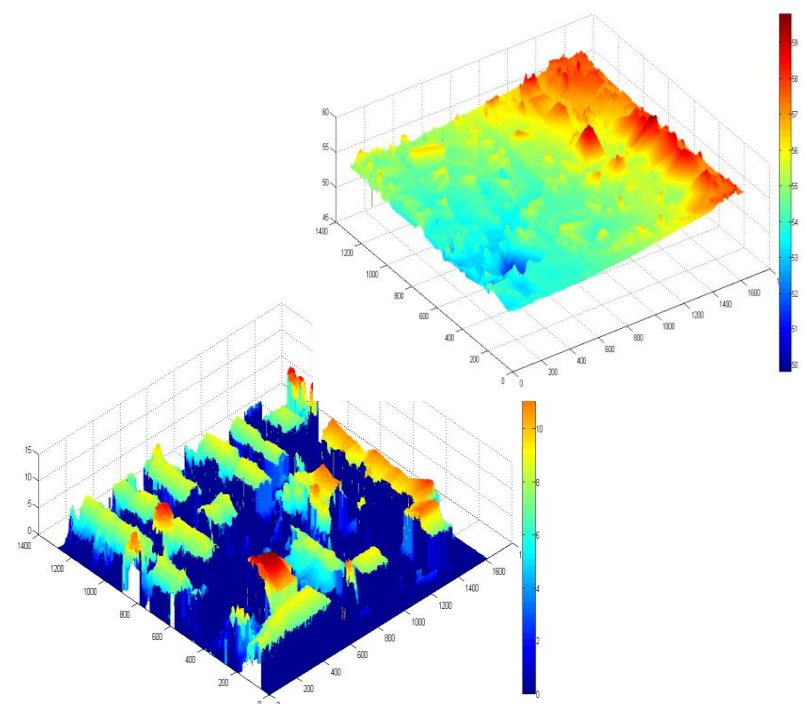

Figure 5 (a)) DTM obtained from the study area) NDSM obtained from the study area

In order to extract features, using simultaneous occurrence matrix features mean, variance, homogeneity, contrast, dissimilarity, entropy, second moment, and correlation for each of the RGB band image Orthophoto area in four directions $0=\theta, 4 / \pi=\theta, 2 / \pi=\theta$ and $4 / \pi 3=\theta$ with a distance $d=1$ were extracted from four matrix obtained were averaged, so each window size in each spectral band, was produced eight features. In order to classify municipal duties, training and assessment as visual examples for each class was prepared by a master of image Orthophoto area. Classes used to classify urban problems, including buildings, trees and tall vegetation, grass and vegetation short, paved road and is impervious surfaces. Class consists of impervious surfaces such as pavement conditions, the cement, the car, the roof is stored. In order to pixel-based classification and selection of optimal features of classification was GASVM pixel basis. GASVM radial function was used in the kernel. As noted, in this study, parameter optimization of SVM, 
feature selection took place at the same time. Interval $\left[2^{\wedge}(-5), 2^{\wedge} 10\right]$ and $\left[2^{\wedge}\right.$ $(-7), 2^{\wedge} 3$, respectively for $\mathrm{C}$ and $\gamma$ parameters were considered. After the implementation GASVM band input of 17 bands were selected as features and optimal parameters $\mathrm{C}$ and $\gamma$ respectively 245 and 775/0 was selected. Figure 7 graph algorithm convergence transgenic best Kappa coefficient based on the best features and kernel parameter indicates the term compensation.

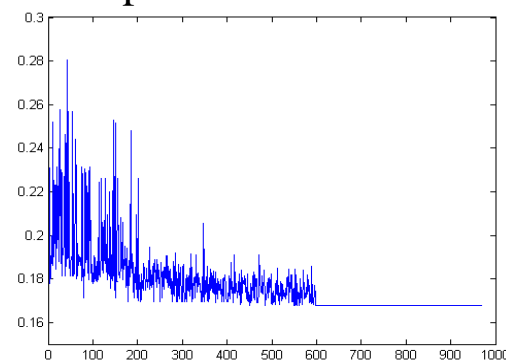

Figure 6 charts the genetic algorithm convergence to the lowest amount of merit (best Kappa coefficient

The evaluation criteria for classification accuracy GASVM Orthophoto prepared from UAV images of the study area is given in Table 1.

Table 1 - Results of the evaluation criteria for classification accuracy provided by GASVM Orthophoto UAV images of the study area

\begin{tabular}{|c|c|c|}
\hline No. & Class name & User Acc. \\
\hline $\mathbf{1}$ & Building & 99.11440881 \\
\hline $\mathbf{2}$ & Trees and tall vegetation & 78.38949843 \\
\hline $\mathbf{3}$ & Grass and short vegetation & 92.8974359 \\
\hline $\mathbf{4}$ & Asphalted road & 91.16179615 \\
\hline $\mathbf{5}$ & Impervious surfaces & 94.89164087 \\
\hline & Overall Accuracy & 90.52422288 \\
\hline \multicolumn{2}{r}{ Kappa Coefficient } & 88.14682948 \\
\hline
\end{tabular}

According to Table 1, the most accurate classification GASVM, for office buildings, and the lowest accuracy of impermeable surfaces, due to the spectral similarity with other classes. In order to achieve the classification results with higher accuracy and spectral composition, texture, and shape conceptual image featureOrthophoto area was fencing. The division of the multiscale-package was used. Because based on the integration of a pixel area which has started and for successive pixels or objects merge the existing image. Parameters of the scale, shape and homogeneity on the basis of resolution Orthophoto and complications in the area, respectively with $30,2.0$ and 3.0 were considered. Figure 7 Orthophoto segmented by segmenting Chndmqyas. $\mathrm{h}$ show.

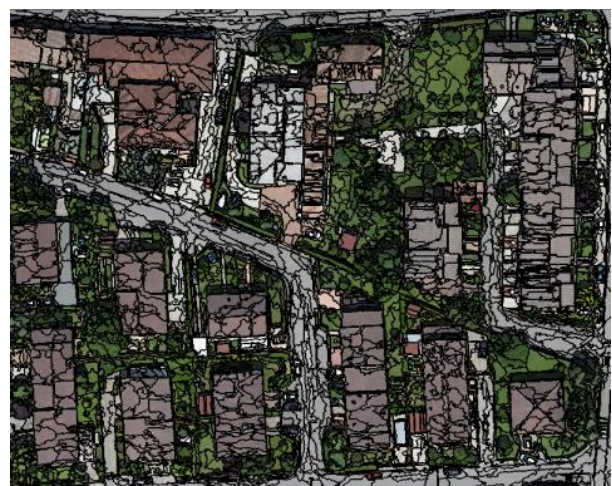

Figure. 7 urban features multi-scale

Segmentation of Ortho photo made from taken images from UAV

Finally, The image acquisition is classified for urban side of the area of integration of the results of the classification of the pixels were GASVM as the segment that more than 50 percent of the class classification were included in the picture belonged to that class..

Table 2 - Results of the evaluation criteria for classification accuracy urban of the integration of the results of classification using multi-scale Orthophoto GASVM and fencing made from UAV images of the study area 


\begin{tabular}{|c|c|c|}
\hline No. & Class name & User Acc. \\
\hline $\mathbf{1}$ & Building & 99.66515188 \\
\hline $\mathbf{2}$ & Trees and tall vegetation & 86.22039135 \\
\hline $\mathbf{3}$ & Grass and short vegetation & 94.6337926 \\
\hline $\mathbf{4}$ & Asphalted road & 93.98350315 \\
\hline $\mathbf{5}$ & Impervious surfaces & 94.22730006 \\
\hline \multicolumn{2}{|c|}{ Overall Accuracy } & 93.47752291 \\
\hline \multicolumn{2}{|c|}{ Kappa Coefficient } & 91.84276828 \\
\hline
\end{tabular}

By comparing the results in Table 1 and 2 is classified as urban feature Orthophoto prepared from UAV images using the integrated multi-scale image classification Bkhsh $\neg$ Bndy GASVM and more accurate classification of all classes to use for single GASVM.

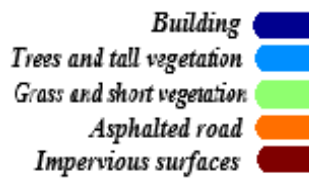

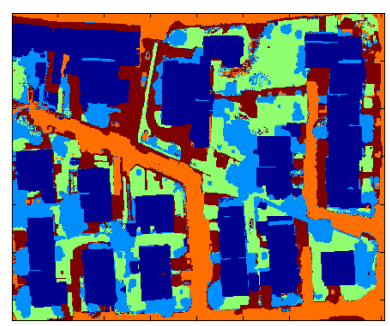

b

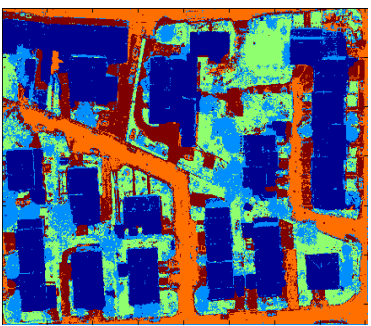

a
Figure. 8 Classification of urban features Orthophoto city derived from images taken from the

UAV images a) classification GASVM b) the integration of multi-scale classification .

\section{Conclusion}

The use of UAVs in the application of photogrammetry to obtain cover images and achieve the main objectives of the photogrammetric mapping has been a boom in the region. Due to the need for such systems in photogrammetric applications, the need for the feasibility of such systems for the classification of urban toll system images are palpable. In this study, using the integration of the results of multi-scale image classification and the classification GASVM urban problems with quality and precision than using single classification of GASVM Orthophoto prepared from UAV images of the study area better result was reached. So that the overall accuracy and kappa coefficient classification image using this method 953/2 and 696/3 percent, respectively. The use of integration and classification of events leading to the use of spectral information, texture and geometric feature and background better classification of urban problems, particularly in Orthophoto UAV images is provided.

\section{REFERENCES}

P. Axelsson, "DEM generation from laser scanner data using adaptive TIN models," International Archives of Photogrammetry and Remote Sensing, vol. 33, pp. 111-118, 2000.

G. Xu and Z. Zhang, Epipolar geometry in stereo, motion and object recognition: a unified approach vol. 6: Springer, 1996.

A. C. Bovik, M. Clark, and W. S. Geisler, "Multichannel texture analysis using localized spatial filters," Pattern Analysis and Machine Intelligence, IEEE Transactions on, vol. 12, pp. 55-73, 1990.

[4] M. Clark, A. C. Bovik, and W. S. Geisler, "Texture segmentation using Gabor modulation/demodulation," Pattern Recognition Letters, vol. 6, pp. 261-267, 1987.

[5] J. G. Daugman, "Complete discrete 2-D Gabor transforms by neural networks for image analysis and compression," Acoustics, Speech and Signal Processing, IEEE Transactions on, vol. 36, pp. 1169-1179, 1988.

[6] H. Gross and U. Thoennessen, "Extraction of lines from laser point clouds," in Symposium of ISPRS Commission III:. 